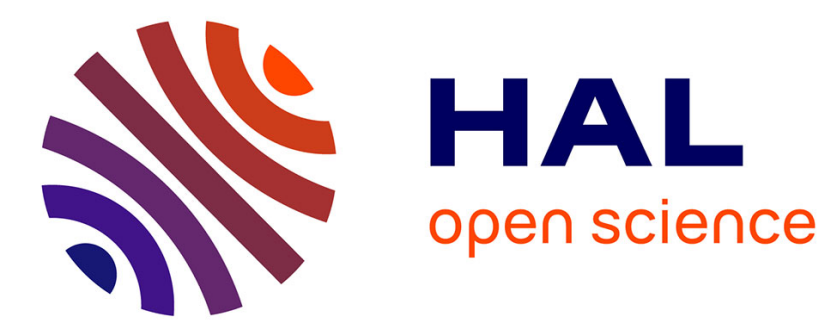

\title{
Qualitative analysis of classes of motion for multiresonant systems I. An algebraic method
}

Angelo Luongo, Angelo Di Egidio, Achille Paolone

\section{To cite this version:}

Angelo Luongo, Angelo Di Egidio, Achille Paolone. Qualitative analysis of classes of motion for multiresonant systems I. An algebraic method. Acta Mechanica, 2005, 174 (1-2), pp.91-107. hal00794609

\section{HAL Id: hal-00794609 \\ https://hal.science/hal-00794609}

Submitted on 26 Feb 2013

HAL is a multi-disciplinary open access archive for the deposit and dissemination of scientific research documents, whether they are published or not. The documents may come from teaching and research institutions in France or abroad, or from public or private research centers.
L'archive ouverte pluridisciplinaire HAL, est destinée au dépôt et à la diffusion de documents scientifiques de niveau recherche, publiés ou non, émanant des établissements d'enseignement et de recherche français ou étrangers, des laboratoires publics ou privés. 


\title{
Qualitative analysis of classes of motion for multiresonant systems I. An algebraic method
}

\author{
A. Luongo, A. Di Egidio, L'Aquila, and A. Paolone, Rome Italy
}

\begin{abstract}
Summary. A general multiresonant system is considered, in which the linear frequency and, possibly, a forcing frequency are involved in a set of linear conditions. The nature of the resonances is first discussed, by distinguishing independent and dependent equations, and both the analysis and design problems of the system are addressed. Rules are then given to construct the qualitative form of the AMEs to any desired order. Two families of terms are identified: improper resonant terms (not associated with any resonance conditions) and proper resonant terms (depending on the specific conditions), sub divided into primary (of lower order) and secondary (of higher order). Theorems are proved to show that both improper and secondary resonant terms have no qualitative but only quantitative effects on classes of motion; reference is therefore made to reduced equations. An algebraic algorithm is illustrated to determine classes of motion, using only the integer resonance coefficients. The concept of degree of constraint of a given resonance condition is introduced, entailing a hierarchic order among the resonance conditions, the implications of which are discussed. Finally, some numerical simulations are shown to test the robustness of classes of motion to higher order terms not accounted for in the asymptotic analysis.
\end{abstract}

\section{Introduction}

It is well known that the asymptotic behavior of resonant systems is captured by a small set of Amplitude Modulation Equations (AMEs), as furnished by the Multiple Scale Method or by equivalent perturbation procedures [1]. These equations govern the time-evolution of the normal mode amplitudes involved in the resonance conditions. One of the most interesting results produced by nonlinear interaction is the existence of so-called (incomplete) classes of motion, i.e., motions in which only a subset of amplitudes is different from zero, while the remaining amplitudes vanish. If non-trivial initial conditions are assigned to the amplitudes of this class only, then the motion develops in a subspace of the state space, which is therefore invariant.

So far, classes of motion have been analyzed in the literature by inspection, after having determined the relevant AMEs. However, in order to perform a qualitative analysis, it would be desirable to use a mathematical tool able to furnish all the existing classes of motion of a nonlinear system, before applying a perturbation method. This paper achieves the objective of illustrating both an algebraic (Part I) and a geometrical (Part II) method to detect classes of motion of resonant systems.

For a general free or harmonically forced multiresonant system the qualitative form (i.e., to within the coeffcients) of the AMEs is given. Several families of terms are classified and their 
role in the evaluation of the classes of motion is discussed. In particular, a reduced form of AMEs able to furnish exhaustive qualitative information is found. Based on this, an algebraic method is proposed to identify all the existing classes of motion. Finally a numerical simulation is presented to analyze how higher-order terms not accounted for in the perturbation analysis affect the classes of motion.

Part II of the paper illustrates an alternative geometrical approach to the problem. The stability of steady solutions is also addressed and the effect of classes of perturbation qualitatively analyzed.

\section{Multiresonant system equations}

The nature of the resonance condition existing in nonlinear dynamical systems is discussed, and the form of the amplitude equations governing the asymptotic motion is then derived.

\subsection{Independent and dependent resonance conditions}

A nonlinear (continuous or discrete) system is in resonance condition when linear combinations with integer coefficients $k_{s n}$ of $N$ natural frequencies $\omega_{n}$ (here assumed to be all different from zero) and of the forcing frequency $\omega_{0}$ are nearly equal to zero, namely,

$\sum_{n \in \mathcal{N}^{+}} k_{s n} \omega_{n}=\sigma_{s}, \quad s=1,2, \ldots, S, \quad k_{s n} \in \mathbb{Z}$,

where $\sigma_{s} \ll 1$ are small detuning parameters and $\mathscr{N}^{+}=\{0,1, \ldots, N\}$. If $\omega_{0}=0$ the resonance is called internal; if $\omega_{0} \neq 0$ it is called external or parametric, according to the nature of the load. In the following, reference will be made to external excitations, although most of the results are believed to apply also to parametric excitations. If $S=1$ the resonance is said to be simple, if $S>1$ it is said to be multiple. The integer

$K_{s}:=\sum_{n \in \mathcal{N}^{+}}\left|k_{s n}\right|-1$

is called the order of the $s$-th resonance condition [2]. It is equal to the degree of nonlinearity producing the resonance for the first time in a perturbation scheme.

In the analysis problem of a system, frequencies $\omega_{n}$ are known, while the coefficients $k_{s n}$ satisfying Eqs. (1) and the total number $S$ of the resonance conditions are unknown. The coefficients can be obtained by applying a brute algorithm (see Appendix A) in which the integers $k_{s n}$ vary within the range $\left[-K_{\max }, K_{\max }\right]$, where $K_{\max }$ is a predefined maximum order of the resonance accounted for. By looking for the linear combinations $\sum_{n \in \mathcal{N}^{+}} k_{s n} \omega_{n}$ which are in absolute value less than or equal to a predefined minimum detuning $\sigma_{\min }=\sigma_{\min }\left(K_{S}\right)$, Eqs. (1) are derived. Naturally, the higher $K_{\max }$ and/or $\sigma_{\min }$, the higher the number $S$ of resonance conditions. However, only $R:=\operatorname{rank}\left[k_{s n}\right]$ conditions are independent, the remaining $Q:=S-R$ conditions being linear combinations of the former. A particular but important case occurs when one condition is proportional to another, i.e., when $k_{q n}=\alpha k_{m n} \forall n$, with $\alpha \neq-1$ integer. The former $R$ equations will be referred to as independent resonance conditions, while the remaining $Q$ equations will be referred to as dependent resonance conditions [3]. In the following it will be shown that, although each of the two sets contributes to the amplitude equations, they occasionally play different roles on the qualitative character of motion. 
In the design problem of a system, it would be desirable to fix, in a given set of frequencies, $R$ independent resonance conditions of orders $K_{s} \leq K_{\max }$ and to exclude, the occurrence of other resonances in the same set up to the order $K_{\max }$. To solve this problem, the following strategy is followed (see Appendix A): first, all the values of the frequencies leading to new independent resonance conditions are detected, and frequencies sufficiently far from the resonant values are then chosen. However, the $R$ independent conditions entail $Q$ unknown dependent conditions; these are determined (see Appendix A) by looking for all the possible resonance conditions of orders $K_{s} \leq K_{\max }(s=R+1, R+2, \ldots, S)$ which do not increase the rank $R$ of the matrix $\left[k_{s n}\right]$. In this way, the whole set of $S$ equations is built up.

As an example of a design problem, if $R=2$ conditions $\omega_{1}=\omega_{2}$ and $\omega_{2}=\omega_{3}$ are enforced in the set $\left\{\omega_{1}, \omega_{2}, \omega_{3}\right\}$, the frequencies are univocally determined once one of them is chosen; moreover, no other independent condition can occur, since the rank of $\left[k_{s n}\right]$ must be less than the number of frequencies (see Appendix A). If $K_{\max }=3$ is fixed, $Q=4$ dependent conditions are found, namely, $\omega_{1}=\omega_{3}, 2 \omega_{1}=\omega_{2}+\omega_{3}, 2 \omega_{2}=\omega_{1}+\omega_{3}$ and $2 \omega_{3}=\omega_{1}+\omega_{2}$; therefore $S=6$ resonant conditions must be accounted for up to the order 3 , two of which are independent and the remaining four dependent. Vice versa, if the three frequencies are found in the analysis problem, $S=6$ conditions would be detected, from which a pair of independent conditions should be selected. In the same example, if $K_{\max }=5$ were taken instead, conditions such as $3 \omega_{1}=2 \omega_{2}+\omega_{3}$ would appear both in the analysis and in the design problem, so that more dependent conditions would be associated with the same independent conditions.

\subsection{Amplitude modulation equations}

According to the Multiple Scale Method [4], the asymptotic behavior of a multiresonant dynamical system is described by

$\mathbf{x}(t)=\sum_{n \in \mathcal{N}^{+}} A_{n}(t) \mathbf{u}_{n} e^{i \omega_{n} t}+$ c.c. + higher-order terms,

where $\mathbf{x}$ is the state vector, $A_{1}, \ldots, A_{N}$ are slowly varying complex amplitude functions of the time $t, \mathbf{u}_{1}, \mathbf{u}_{2}, \ldots, \mathbf{u}_{N}$ are the linear modes associated with the frequencies $\omega_{n}, A_{0}=$ const. is the excitation amplitude and $\mathbf{u}_{0}$ the excitation spatial shape. The amplitude evolution is governed by the so-called Amplitude Modulation Equations (AMEs), obtained by zeroing the resonant terms in any perturbation equation and by collecting them according to the reconstitution procedure [5]. The form of the AMEs depends only on the resonant conditions (1), so that it can be predicted before expanding and solving the perturbation equations, according to the following procedure [3].

In $K$-th order perturbation equations nonlinearities of order $K$ and lower are present. However, they all produce terms which are monomials $\mathbf{p}_{K}$ of degree $K$, whose factors are $A_{n} e^{i \omega_{n} t}$ and $\overline{A_{n}} e^{i \omega_{n} t}$, namely:

$\mathbf{p}_{K}:=\left\{\left(\prod_{n \in \mathcal{N}^{ \pm}} A_{n}^{l_{n}}\right) \exp \left[i \sum_{n \in \mathcal{N}^{+}}\left(l_{n}-l_{n}\right) \omega_{n} t\right] \mid \sum_{n \in \mathcal{N}^{ \pm}} l_{n}=K, l_{n} \in \mathbb{N}\right\}$,

where $A_{n}:=\overline{A_{n}}(n=0,1, \ldots, N)$ and $\mathscr{N}^{ \pm}=\{ \pm 0, \pm 1, \ldots, \pm N\}$, by formally distinguishing \pm 0 . These terms cause resonance on the $m$-th mode (and must therefore be moved to the $m$-th $\mathrm{AME}$ ) if and only if their frequencies are nearly equal to $\omega_{m}$, as given by whichever of the resonance conditions (1), both of independent and dependent type. Now, Eqs. (1) can be solved for $\omega_{m}$, for all $s \in[0, S]$ so that $k_{s m} \neq 0$, namely: 
$\omega_{m}=\sum_{n \in \mathcal{N}^{+}} k_{s m n} \omega_{n} \pm \sigma_{s}, \quad k_{s m n}:=\mp k_{s n}+\delta_{m n}$,

where the upper sign must be taken if $k_{s m}>0$, the lower sign if $k_{s m}<0$ and $\delta_{m n}$ is the Kronecker symbol. Therefore, from Eqs. (4) and (5) all the elements of $\mathbf{p}_{K}$ in which $l_{n}-l_{n}=k_{s m n} \forall n \in \mathscr{N}^{+}$are resonant, of frequency $\omega_{m} \mp \sigma_{s}$. They will be referred to as proper resonant terms; in general one or more of these terms are found at the $K$-th order for each resonance condition of order $K_{s} \leq K$.

In addition to the previous terms, others such as

$A_{m}, A_{m}\left(A_{i} \bar{A}_{i}\right)^{k_{i}}, A_{m}\left(A_{i} \bar{A}_{i}\right)^{k_{i}}\left(A_{j} \bar{A}_{j}\right)^{k_{j}}, \ldots, \quad(i, j) \in \mathscr{N}^{+},\left(k_{i}, k_{j}\right)=1,2, \ldots$,

produced by small damping and odd nonlinearities, must be removed from the right hand member of the perturbation equations in order to allow solvability. Since they are not all associated with resonance conditions, they will be referred to as improper resonant terms. Moreover, as a special case, if two (or more) frequencies are coincident, e.g., $\omega_{i}=\omega_{j}$, additional terms of the type $A_{m}\left(A_{i} \bar{A}_{j}\right)^{k_{i}}, A_{m}\left(A_{j} \bar{A}_{i}\right)^{k_{j}}$ with $i \neq j$ must be accounted for.

In conclusion the AMEs read [3]

$A_{m}^{\prime}=\mathscr{L}_{m}\left(A_{m}, A_{m}\left(A_{n} \bar{A}_{n}\right), \ldots, \prod_{n \in \mathcal{N}^{ \pm}} A_{n}^{l_{\text {smn }}} e^{\mp i \sigma_{s} t}, \ldots\right) m=1,2, \ldots, N$,

in which $\mathscr{L}_{m}$ is a complex linear operator with constant coefficients and the exponents $l_{s m n}$ are (generally not unique) solutions of:

$l_{s m n}-l_{s m, n}=k_{s m n} \forall n \in \mathscr{N}^{+}, \quad \sum_{n \in \mathscr{N}^{ \pm}} l_{s m n}=K, l_{s m n} \in \mathbb{N}, K \in\left[K_{s}, K_{\max }\right]$.

Problem (8) is solved as follows. At the lowest order $K=K_{s}$, the solution $l_{s m n}=l_{s m n}^{0}$ and $l_{s m, n}=l_{s m, n}^{0}$ holds, with

$l_{s m n}^{0}:=\left(k_{s m n}, 0,0\right), \quad l_{s m, n}^{0}:=\left(0,0, k_{s m n}\right)$ for $\left(k_{s m n}>0, k_{s m n}=0, k_{s m n}<0\right)$.

This solution will be called the generating solution; it is unique at order $K_{s}$, since, in order to satisfy Eqs. (8.1), both $l_{s m n}^{0}$ and $l_{s m, n}^{0}$ should be incremented by the same quantity, thus violating Eqs. (8.2). Other solutions (called generated solutions) are instead found at the orders $K_{s}+\Delta K$, when $\Delta K$ is even, of the form

$l_{s m n}=l_{s m n}^{0}+h_{n}, \quad l_{s m, n}=l_{s m, n}^{0}-h_{n}, \quad \sum_{n \in \mathcal{N}^{+}} h_{n}=\Delta K / 2, \quad h_{n} \in \mathbb{N}$,

while no solutions exist when $\Delta K$ is odd. To sum up, a resonance of order $K_{s}$ produces terms in the AME at the orders $K=K_{s}, K_{s}+2, K_{s}+4, \ldots$ At the $K_{s}$-th order there is a unique generating resonant term $\mathscr{A}_{s m} \exp \left(\mp i \sigma_{s} t\right)$ with

$\mathscr{A}_{s m}:=\prod_{n \in \mathscr{N}^{ \pm}} A_{n}^{l_{s m n}^{0}}$

associated with solution (9); at higher orders there are more generated resonant terms:

$\mathscr{A}_{s m}\left(A_{i} \bar{A}_{i}\right)^{k_{i}}, \quad \mathscr{A}_{s m}\left(A_{i} \bar{A}_{i}\right)^{k_{i}}\left(A_{j} \bar{A}_{j}\right)^{k_{j}}, \ldots, \quad(i, j) \in \mathscr{N}^{+},\left(k_{i}, k_{j}\right)=1,2, \ldots$,

associated with solutions (10). These can be obtained by recursively multiplying $\mathscr{A}_{\text {sm }}$ by $\left(A_{i} \bar{A}_{i}\right)$. The similarity among the nonlinear improper resonant terms (6) and the generated resonant terms (12) should be noticed. 
The resonant terms of the lowest order will be called the primary resonant terms; the terms of higher orders will be called the secondary resonant terms. Usually they are given by Eqs. (11) and (12), respectively, but exceptions to this rule exist, as will be explained below.

As an example, if the order- 2 resonance condition $\omega_{i}=2 \omega_{j}$ is considered, the primary resonant term in the $A_{i}$-equation is $A_{j}^{2}$, which appears at the order $K=2$; at the order $K=4$ it generates the secondary terms $A_{j}^{3} \bar{A}_{j}$ and $A_{i} \bar{A}_{i} A_{j}^{2}$. Moreover, by writing the resonance condition as $\omega_{j}=\omega_{i}-\omega_{j}$, the primary term in the $A_{j}$-equation is $A_{i} \bar{A}_{j}$ and the secondary terms are $A_{i} A_{j} \bar{A}_{j}^{2}$ and $A_{i}^{2} \bar{A}_{i} \bar{A}_{j}$. If the analysis is taken one step further, no new generated terms arise at the order $K=5$. However, since the (dependent) condition $2 \omega_{i}=4 \omega_{j}$ exists at this order, resonant terms $A_{j}^{4} \bar{A}_{i}$ and $A_{i}^{2} \bar{A}_{j}^{3}$ must be considered in the $A_{i}$ - and $A_{j}$-equations, respectively. Strictly speaking, these terms are generating terms for the dependent condition; however, due to the nature of such a condition (it is simply proportional to the original one), it is more suitable to classify them as added generated terms.

Examples of the AMEs for systems in simple $(S=1)$ internal or external resonance are given in Tables 1 and 2, respectively. Only additive resonances have been considered in the Tables; subtractive resonances (e.g., $\omega_{i}=\omega_{j}-\omega_{k}$ ) can be obtained from the former by exchanging the relevant complex amplitudes with their conjugate (i.e., $A_{k}$ with $\overline{A_{k}}$ in the example). In these, the form of the relevant AMEs is shown with the primary terms highlighted in bold characters. Harmonic dependence on time, due to the detunings in Eqs. (7), has not been made explicit, since it is unnecessary to the qualitative discussion developed in the following. Therefore, from now on operators $\mathscr{L}_{m}$ must be understood as having coefficients harmonically varying in time. The first cases in Tables 1 and 2 are associated with quadratic nonlinearities $\left(K_{s}=2\right)$; the last cases with cubic nonlinearities $\left(K_{s}=3\right)$. However, if nonlinearities of higher degree are accounted for, more terms arise in the equation, as discussed. Special cases occur for the

Table 1. AMEs for internally resonant systems

\section{Order 2 resonances}

\begin{tabular}{cccc}
\hline$\omega_{i}$ & $2 \omega_{j}$ & $A_{i}^{\prime}$ & $\mathscr{L}_{i}\left(A_{i} ; \mathbf{A}_{j}^{2}\right)$ \\
& & $A_{j}^{\prime}$ & $\mathscr{L}_{j}\left(A_{j} ; \mathbf{A}_{i} \mathbf{A}_{j}\right)$ \\
$\omega_{i}$ & $\omega_{j}+\omega_{k}$ & $A_{i}^{\prime}$ & $\mathscr{L}_{i}\left(A_{i} ; \mathbf{A}_{j} \mathbf{A}_{k}\right)$ \\
& & $A_{j}^{\prime}$ & $\mathscr{L}_{j}\left(A_{j} ; \mathbf{A}_{i} \mathbf{A}_{k}\right)$ \\
& & $A_{k}^{\prime}$ & $\mathscr{L}_{k}\left(A_{k} ; \mathbf{A}_{i} \mathbf{A}_{j}\right)$
\end{tabular}

Order 1 resonance

$\omega_{i} \quad \omega_{j}$

$$
\begin{array}{ll}
A_{i}^{\prime} & \mathscr{L}_{i}\left(A_{i}, A_{i}^{2} A_{i}, A_{i} A_{j} A_{j} ; \mathbf{A}_{i} \mathbf{A}_{i} \mathbf{A}_{j}, \mathbf{A}_{j}^{2} \mathbf{A}_{j}, \mathbf{A}_{j}^{2} \mathbf{A}_{i}\right) \\
A_{j}^{\prime} & \mathscr{L}_{j}\left(A_{j} ; A_{j}^{2} A_{j}, A_{j} A_{i} A_{i} ; \mathbf{A}_{j} \mathbf{A}_{j} \mathbf{A}_{i}, \mathbf{A}_{i}^{2} \mathbf{A}_{i}, \mathbf{A}_{i}^{2} \mathbf{A}_{j}\right)
\end{array}
$$

Order 3 resonances
$\omega_{i} \quad 3 \omega_{j}$
$\omega_{i} \quad 2 \omega_{j}+\omega_{k}$
$\begin{array}{ll}A_{i}^{\prime} & \mathscr{L}_{i}\left(A_{i} ; A_{i}^{2} A_{i}, A_{i} A_{j} A_{j} ; \mathbf{A}_{j}^{3}\right) \\ A_{j}^{\prime} & \mathscr{L}_{j}\left(A_{j} ; A_{j}^{2} A_{j}, A_{j} A_{i} A_{i} ; \mathbf{A}_{i} \mathbf{A}_{j}^{2}\right)\end{array}$
$A_{i}^{\prime} \quad \mathscr{L}_{i}\left(A_{i} ; A_{i}^{2} A_{i}, A_{i} A_{j} A_{j}, A_{I} A_{k} A_{k} ; \mathbf{A}_{i} \mathbf{A}_{j} \mathbf{A}_{k}\right)$
$A_{j}^{\prime} \quad \mathscr{L}_{j}\left(A_{j} ; A_{j}^{2} A_{j}, A_{j} A_{i} A_{i}, A_{j} A_{k} A_{k} ; \mathbf{A}_{i}^{2} \mathbf{A}_{k}\right)$
$A_{k} \quad \mathscr{L}_{k}\left(A_{k} ; A_{k}^{2} A_{k}, A_{k} A_{i} A_{i}, A_{k} A_{j} A_{j} ; \mathbf{A}_{i}^{2} \mathbf{A}_{j}\right)$
$\omega_{i} \omega_{j}+\omega_{h}+\omega_{k}$
$A_{i}^{\prime} \quad \mathscr{L}_{i}\left(A_{i} ; A_{i}^{2} A_{i}, A_{i} A_{j} A_{j}, A_{i} A_{h} A_{h}, A_{i} A_{k} A_{k} ; \mathbf{A}_{j} \mathbf{A}_{h} \mathbf{A}_{k}\right)$
$A_{j}^{\prime} \quad \mathscr{L}_{j}\left(A_{j} ; A_{j}^{2} A_{j}, A_{j} A_{i} A_{i}, A_{j} A_{h} A_{h}, A_{j} A_{k} A_{k} ; \mathbf{A}_{i} \mathbf{A}_{h} \mathbf{A}_{k}\right)$
$A_{h}^{\prime} \quad \mathscr{L}_{j}\left(A_{h} ; A_{h}^{2} A_{h}, A_{h} A_{i} A_{i}, A_{h} A_{j} A_{j}, A_{h} A_{k} A_{k} ; \mathbf{A}_{i} \mathbf{A}_{j} \mathbf{A}_{k}\right)$
$A_{k}^{\prime} \quad \mathscr{L}_{k}\left(A_{k} ; A_{k}^{2} A_{k}, A_{k} A_{i} A_{i}, A_{k} A_{j} A_{j}, A_{k} A_{h} A_{h} ; \mathbf{A}_{i} \mathbf{A}_{j} \mathbf{A}_{h}\right)$


Table 2. AMEs for internally and externally resonant systems

Order 2 resonances

\begin{tabular}{llll}
\hline$\omega_{0}$ & $2 \omega_{i}$ & $A_{i}^{\prime}$ & $\mathscr{L}_{i}\left(A_{i} ; \mathbf{A}_{0} \mathbf{A}_{i}\right)$ \\
$2 \omega_{0}$ & $\omega_{i}$ & $A_{i}^{\prime}$ & $\mathscr{L}_{i}\left(A_{i} ; \mathbf{A}_{0}^{2}\right)$ \\
$\omega_{0}$ & $\omega_{i}+\omega_{j}$ & $A_{i}^{\prime}$ & $\mathscr{L}_{i}\left(A_{i} ; \mathbf{A}_{0} \mathbf{A}_{j}\right)$ \\
& & $A_{j}^{\prime}$ & $\mathscr{L}_{j}\left(A_{j} ; \mathbf{A}_{0} \mathbf{A}_{j}\right)$ \\
\hline
\end{tabular}

Order 1 resonance

$\omega_{0} \quad \omega_{i} \quad A_{i}^{\prime} \quad \mathscr{L}_{i}\left(A_{i} ; A_{i}^{2} A_{i} ; \mathbf{A}_{0}\right)$

\section{Order 3 resonances}

\begin{tabular}{lllll}
\hline$\omega_{0} \quad 3 \omega_{i}$ & $A_{i}^{\prime}$ & $\mathscr{L}_{i}\left(A_{i} ; A_{i}^{2} A_{i}, A_{i} A_{0} A_{0} ; \mathbf{A}_{0} \mathbf{A}_{i}^{2}\right)$ \\
$3 \omega_{0}$ & $\omega_{i}$ & $A_{i}^{\prime}$ & $\mathscr{L}_{i}\left(A_{i} ; A_{i}^{2} A_{i}, A_{i} A_{0} A_{0} ; \mathbf{A}_{0}^{3}\right)$ \\
$\omega_{0} \quad 2 \omega_{i}+\omega_{j}$ & $A_{i}^{\prime}$ & $\mathscr{L}_{i}\left(A_{i} ; A_{i}^{2} A_{i}, A_{i} A_{j} A_{j}, A_{i} A_{0} A_{0} ; \mathbf{A}_{0} \mathbf{A}_{i} \mathbf{A}_{j}\right)$ \\
& & $A_{j}^{\prime}$ & $\mathscr{L}_{j}\left(A_{j} ; A_{j}^{2} A_{j}, A_{j} A_{i} A_{i}, A_{j} A_{0} A_{0} ; \mathbf{A}_{0} \mathbf{A}_{i}^{2}\right)$ \\
$\omega_{i} \quad 2 \omega_{0}+\omega_{j}$ & $A_{i}^{\prime}$ & $\mathscr{L}_{i}\left(A_{i} ; A_{i}^{2} A_{i}, A_{i} A_{j} A_{j}, A_{i} A_{0} A_{0} ; \mathbf{A}_{0}^{2} \mathbf{A}_{j}\right)$ \\
& & $A_{j}^{\prime}$ & $\mathscr{L}_{j}\left(A_{j} ; A_{j}^{2} A_{j}, A_{j} A_{i} A_{i}, A_{j} A_{0} A_{0} ; \mathbf{A}_{i} \mathbf{A}_{0}^{2}\right)$ \\
$\omega_{0} \quad \omega_{i}+\omega_{j}+\omega_{k}$ & $A_{i}^{\prime}$ & $\mathscr{L}_{i}\left(A_{i} ; A_{i}^{2} A_{i}, A_{i} A_{j} A_{j}, A_{i} A_{k} A_{k}, A_{i} A_{0} A_{0} ; \mathbf{A}_{0} \mathbf{A}_{j} \mathbf{A}_{k}\right)$ \\
& & $A_{j}^{\prime}$ & $\mathscr{L}_{j}\left(A_{j} ; A_{j}^{2} A_{j}, A_{j} A_{i} A_{i}, A_{j} A_{k} A_{k}, A_{j} A_{0} A_{0} ; \mathbf{A}_{0} \mathbf{A}_{i} \mathbf{A}_{k}\right)$ \\
& & $A_{k}^{\prime}$ & $\mathscr{L}_{k}\left(A_{k} ; A_{k}^{2} A_{k}, A_{k} A_{i} A_{i}, A_{k} A_{j} A_{j}, A_{k} A_{0} A_{0} ; \mathbf{A}_{i} \mathbf{A}_{i} \mathbf{A}_{j}\right)$
\end{tabular}

order-1 resonances $\omega_{i}=\omega_{j}$ and $\omega_{i}=\omega_{0}$. Indeed, these resonances cannot manifest at order 1 , since the relevant perturbation equations are linear (a peculiarity of the perturbation method) and homogeneous (since the excitation must be assumed to be of a soft type in order to avoid divergence, i.e., $A_{0}=O\left(A_{i}^{3}\right)$ [4]). Consequently, the resonances occur at a higher order, namely $K=3$. In the $\omega_{i}=\omega_{j}$ case, the generating terms $A_{j}$ and $A_{i}$ do not appear at order 1; however, according to Eqs. (12), they generate at order 3 the terms $\left(A_{i} \overline{A_{i}} A_{j}, A_{j}^{2} \overline{A_{j}}\right)$ and $\left(A_{j} \overline{A_{j}} A_{i}, A_{i}^{2} \overline{A_{i}}\right)$, respectively. Moreover, since the dependent condition $2 \omega_{i}=2 \omega_{j}$ also exists at the third order, the terms $A_{j}^{2} \overline{A_{i}}$ and $A_{i}^{2} \overline{A_{j}}$ enter in the two equations, respectively (see Table 1). All these terms, which are firstly responsible for the resonance in the perturbation procedure, are the primary resonant terms of the $\omega_{i}=\omega_{j}$ resonance; they are therefore printed in bold characters in Table 1 . In the $\omega_{i}=\omega_{0}$ case the primary term $A_{0}$ does not appear at order 1 , but only at order 3 , where it has been shifted. Terms as $A_{0} A_{i} \overline{A_{i}}$ are of higher order and have therefore not been accounted for in Table 2.

Only the generic case has been considered in Tables 1 and 2; nongeneric cases in which some terms disappear, e.g. for the existence of symmetry properties, must be analyzed specifically. Thus, if nonlinearities from which the generating terms arise are not present in the equations, a resonance of order $K_{s}$ manifests itself only at a higher order, through a mechanism identical to that discussed before for the 1:1 resonances.

To sum up, it is convenient to classify the proper resonant terms as primary and secondary terms, rather than generating and generated. The primary resonant terms are the proper resonant terms of the lowest order: they usually appear at the order $K_{s}$, equal to that of the resonance (i.e., they are generating terms); however, in the $\omega_{i}=\omega_{j}$ case and in all cases in which nonlinearities are lacking, they appear at a higher order than that of the resonance (i.e., they are generated terms). Even in these cases, the higher-order terms are given by Eqs. (12), with $\mathscr{A}_{s m}$ now being the lower-order existing terms; in addition, added generated terms can come out. 
If multiple $(S>1)$ resonances exist in the system, the relevant AMEs are obtained according to the following general rules: (1) all the improper resonant terms of Eqs. (6) (i.e., for $(i, j) \in \mathscr{N}^{+}$) must be taken in each equation; (2) the proper resonant terms produced by the simple resonances must be summed up in the homonymous equations. The AMEs therefore read:

$A_{m}^{\prime}=\sum_{s}^{S} \mathscr{L}_{m s}(\cdot)+$ improper resonant terms $m=1,2, \ldots, N$,

where $\mathscr{L}_{m s}(\cdot)$ are the proper terms produced by the $s$-th simple resonance. As special cases, the following rule also holds: (3) if secondary terms must be accounted for the $s$-th resonance, all terms (12) must be taken in $\mathscr{L}_{m s}(\cdot)$, even if some amplitudes are not involved in the $s$-th resonance. Resonances of the $\omega_{i}=\omega_{j}$ type, incomplete equations of motion or higher-order approximations call for rule (3). The example below illustrates the procedure.

\subsection{Example}

As an example, a system is considered in which $N=5$ frequencies and the forcing frequency are involved in $R=4$ independent resonance conditions

$\omega_{1}=2 \omega_{2}, \quad \omega_{2}=\omega_{3}+2 \omega_{5}, \quad \omega_{3}=\omega_{4}, \quad \omega_{0}=\omega_{1}$,

the first three being internal, the last external. From Eq. (14), the following $Q=4$ dependent conditions are drawn:

$\omega_{2}=\omega_{4}+2 \omega_{5}, \quad \omega_{0}=2 \omega_{2}, \quad \omega_{0}=\omega_{1} \pm \omega_{3} \mp \omega_{4}$,

so that the resonance conditions to be accounted for are $S=8$. By using the results in Tables 1 and 2 and the law (13), up to order 3 the AMEs read:

$$
\begin{aligned}
& A_{1}^{\prime}=\mathscr{L}_{1}\left(A_{1}, A_{1} A_{1} \overline{A_{1}}, A_{1} A_{2} \overline{A_{2}}, \ldots ; \mathbf{A}_{2}^{2}, \mathbf{A}_{0} ; \mathbf{A}_{0} \mathbf{A}_{3} \overline{\mathbf{A}}_{4}, \ldots\right), \\
& A_{2}^{\prime}=\mathscr{L}_{2}\left(A_{2}, A_{2} A_{1} \overline{A_{1}}, A_{2} A_{2} \overline{A_{2}}, \ldots ; \mathbf{A}_{1} \overline{\mathbf{A}}_{2}, \mathbf{A}_{5} \mathbf{A}_{3}^{2} ; \mathbf{A}_{5}^{2} \mathbf{A}_{4}, \ldots\right), \\
& A_{3}^{\prime}=\mathscr{L}_{3}\left(A_{3}, A_{3} A_{1} \overline{A_{1}}, A_{3} A_{2} \overline{A_{2}}, \ldots ; \mathbf{A}_{2} \overline{\mathbf{A}}_{5}^{2}, \mathbf{A}_{3} \overline{\mathbf{A}}_{3} \mathbf{A}_{4}, \mathbf{A}_{4}^{2} \overline{\mathbf{A}}_{4}, \overline{\mathbf{A}}_{3} \mathbf{A}_{4}^{2} ; \mathbf{A}_{0} \overline{\mathbf{A}}_{1} \mathbf{A}_{4}, \ldots\right), \\
& A_{4}^{\prime}=\mathscr{L}_{4}\left(A_{4}, A_{4} A_{1} \overline{A_{1}}, A_{4} A_{2} \overline{A_{2}}, \ldots ; \mathbf{A}_{4} \overline{\mathbf{A}}_{4} \mathbf{A}_{3}, \mathbf{A}_{3}^{2} \overline{\mathbf{A}}_{3}, \mathbf{A}_{3}^{2} \overline{\mathbf{A}}_{4} ; \overline{\mathbf{A}}_{5}^{2} \mathbf{A}_{2}, \ldots\right), \\
& A_{5}^{\prime}=\mathscr{L}_{5}\left(A_{5}, A_{5} A_{1} \overline{A_{1}}, A_{5} A_{2} \overline{A_{2}}, \ldots ; \mathbf{A}_{2} \overline{\mathbf{A}}_{3} \overline{\mathbf{A}}_{5} ; \mathbf{A}_{2} \overline{\mathbf{A}}_{4} \overline{\mathbf{A}}_{5}\right),
\end{aligned}
$$

where semicolons devide improper, proper independent and proper dependent terms. According to rule (3) of the previous subsection, in the $A_{3}$ - and $A_{4}$-equations all the secondary terms have been taken.

\section{Classes of motion}

Classes of motion are formally defined and some fundamental theorems helpful to their evaluation are proved.

\subsection{Definitions}

When a system oscillates at a steady or unsteady state, all the resonant modes generally contribute to the motion, i.e., all the amplitudes $A_{n}$ are different from zero. However, 
depending on the type of the resonance (and on the initial conditions), some of the amplitudes are allowed to vanish, so that the system oscillates in a smaller number of modes. Such motions will be referred to as (incomplete) classes of motion. From a geometrical point of view, the trajectories of complete motions belong to the whole $2 N$-dimensional state-space $\left(\Re\left(A_{n}\right), \Im\left(A_{n}\right)\right)$, whereas incomplete motions only belong to subspaces.

Formally, a class of motion $\mathscr{C}$ is defined as follows:

$$
\mathscr{C}=\left\{A_{i}, i \in \mathscr{M} \mid A_{j}=0 \forall j \notin \mathscr{M} \Rightarrow \mathscr{L}_{j} \equiv 0 \forall A_{i}\right\},
$$

$\mathscr{M}=\left\{i_{1}, i_{2}, \ldots, i_{M}\right\} \quad M \leq N$.

In words: a class $\mathscr{C}$ is a set of amplitudes $A_{i}$ such that, by zeroing in the AMEs for all the amplitudes $A_{j}$ not included in the set, the associated right-hand members $\mathscr{L}_{j}$ identically vanish for all values of the amplitudes of the class. Therefore, if nontrivial initial conditions are imposed only on the amplitudes of the class, then only such amplitudes participate in the motion. In other words, the amplitudes of a class span an invariant subspace of the state-space. In the following the locution $M$-class will be used to describe a class of $M$ amplitudes. If the system is forced, since $A_{0}$ cannot be made equal to zero by initial conditions, then $A_{0}$ necessarily belongs to all the existing classes.

Classes of (periodic) motions comprising a unique amplitude (the so-called monomodal solutions) are also known as nonlinear normal modes and have been extensively studied for nonresonant systems [6]. Classes comprising one or more amplitudes (multimode solutions) could be considered as a generalization for resonant systems of such nonlinear modes.

To analyze the system's behavior, it would be worth while to determine all the existing classes of motion. A procedure is illustrated here to address this problem qualitatively.

\subsection{Reduced equations of motion}

In order to simplify the search for the classes of motion of a resonant system, the role of the different terms entering the AMEs is studied. The objective of the analysis is to ascertain the existence of reduced equations in which some terms are omitted, and which are still able to give all the qualitative information concerning the complete system. The following lemma is helpful to identify such a reduced system:

Lemma 1: If new terms are entered in a set of equations $A_{m}^{\prime}=\mathscr{L}_{m}^{*}(\cdot)$ to change it into $A_{m}^{\prime}=\mathscr{L}_{m}(\cdot)$, no new classes can arise. If the additional terms do not disrupt any of the existing classes $\mathscr{C}^{*}$ of $\mathscr{L}^{*}$, then they are unessential to the evaluation of the classes $\mathscr{C}$ of $\mathscr{L}$.

Proof: If the new terms, evaluated at $\mathscr{C}^{*}$, all disappear in the vanishing equations (i.e., if $\mathscr{L}_{j}$, in addition to $\mathscr{L}_{j}^{*}$, also vanishes when $A_{j}=0 \forall j \notin \mathscr{M}$, see Eq. (17)) then $\mathscr{C}^{*}$ still survives, otherwise it is disrupted. Moreover, no new classes can arise, since the additional terms, being different, cannot balance the pre-existing nonzero terms. From this follows the second part of Lemma 1.

First of all, the rule of the improper resonant terms (i.e., terms not associated with any resonance conditions) is examined. It is interesting to note that if $S=0$, i.e., if the system is non-resonant, all subsets of $\left\{A_{n}\right\}$ are classes of motion. This property follows from the fact 
that the $m$-th AME contains in this case only the improper terms (Eq. (6)), which are all proportional to $A_{m}$; therefore, the definition (Eq. (17)) holds for any choice of $\mathscr{M}$. This circumstance corroborates the hypothesis that the classes of motion are intrinsically connected to the resonance conditions.

When a resonant system is instead considered (i.e., $S \neq 0$ ), the role of the improper terms is stated by the following theorem:

Theorem 1: The improper resonant terms are unessential in evaluating the classes of motion.

Proof: Let $\mathscr{C}^{*}$ be a class of motion determined by reduced equations $A_{m}^{\prime}=\mathscr{L}_{m}^{*}(\cdot)$ in which only proper resonant terms have been retained; therefore, by Eq. (17), $A_{j}=0 \forall j \notin \mathscr{M}$ entails $\mathscr{L}_{j}^{*}=0$. But $A_{j}=0$ implies that all the improper terms (Eq. (6)) in the $j$-th $\operatorname{AME~}(j \notin \mathscr{M})$ identically vanish, so that also $\mathscr{L}_{j}=0$; therefore $\mathscr{C}^{*}$ is a class of motion even for the complete system. The thesis follows from Lemma 1.

It should be noted that although improper terms are unessential to the existence of classes of motion, they generally affect the motion itself. Therefore, improper terms have a quantitative rather than a qualitative influence on the motion.

By focusing the attention on proper resonant terms, the role of primary and secondary terms is now examined. The following Theorem, similar to Theorem 1, holds:

Theorem 2: The secondary resonant terms are unessential in evaluating the classes of motion.

Proof: Let $\mathscr{C}^{*}$ be a class of motion determined by reduced equations $A_{m}^{\prime}=\mathscr{L}_{m}^{*}\left(\mathscr{A}_{s m}\right)$ in which only primary resonant terms $\mathscr{A}_{s m}(s=1,2, \ldots, S)$ have been retained; therefore, by Eq. (17), $\mathscr{L}_{j}^{*}\left(\mathscr{A}_{s j}\right)=0 \forall j \notin \mathscr{M}$ and $\forall A_{i} \in \mathscr{C}^{*}$. Since the primary terms $\mathscr{A}_{s j}$ in each equation are all independent, it follows that $\mathscr{A}_{s j}=0(s=1,2, \ldots, S) \forall j \notin \mathscr{M}$. Now, by remembering Eq. (12), all the secondary terms in the $j$-th AME are proportional to the vanishing $\mathscr{A}_{s j}$ 's, so that they vanish too. Similar arguments hold if added generated terms also proportional to $\mathscr{A}_{s j}$ are present. Consequently $\mathscr{C}^{*}$ is not disrupted by the secondary terms and by virtue of Lemma 1 , the thesis is proved.

Comments similar to those for relevant improper terms hold for secondary terms; in particular, it follows from Theorem 2 that:

Corollary 1: Asymptotic approximations of an order higher than the order at which all the primary terms have appeared do not modify the classes of motion of a resonant system unless new resonances emerge.

Therefore, higher-order approximations only modify the quantitative characters of motion if no new resonances are encountered. In contrast, if higher-order resonances arise in the given set of frequencies, then some of the lower-order classes could disappear. A numerical investigation of this aspect will be performed in Sect. 4.2.

From Theorems 1 and 2 it ensues that the classes of motion of a system $A_{m}^{\prime}=\mathscr{L}_{m}(\cdot)$ can be determined by reduced equations $A_{m}^{\prime}=\mathscr{L}_{m}^{*}(\cdot)$ in which only primary resonant terms are retained. Therefore, in the following, reference will be made to the reduced system, and the asterisk will be dropped. 


\subsection{An algorithm for evaluating classes of motion}

A practical method to evaluate all the classes of motion admitted by a multiresonant system is now illustrated. It is based on the following theorem:

Theorem 3: A set of amplitudes $\mathscr{C}=\left\{A_{i}\right\} i \in \mathscr{M}$ (necessarily including $A_{0}$, if it is different from zero) is a class of motion for the multiresonant system (1) if and only if

$\sum_{j / \mathscr{M}}\left|k_{s j}\right| \neq 1 \quad \forall s$

Proof: Equation (18) entails that, for any resonance condition, all the $\omega_{h}(h \notin \mathscr{M})$ are a linear combination of frequencies in which at least frequency $\omega_{j}(j \in \mathscr{M})$ appears, possibly in addition to frequencies $\omega_{i}(i \in \mathscr{M})$. By remembering the nature of the primary resonant terms, it follows that all the associated $\mathscr{L}_{h}(\cdot)$ contain impure monomials in the amplitude $\left\{A_{i}\right\}$ (i.e., products of $A_{i}$ and $A_{j}$-amplitudes), and therefore identically vanish for $A_{j}=0(j \notin \mathscr{M})$; hence, $\mathscr{C}$ is a class of motion. Vice versa, if Eq. (18) is not satisfied, at least one resonance condition exists in which $\left|k_{s h}\right|=1, k_{s j}=0(j \neq h, j \notin \mathscr{M})$; therefore a frequency $\omega_{h}$ exists which is a linear combination of frequencies $\omega_{i}(i \in \mathscr{M})$ only. The relevant $\mathscr{L}_{h}(\cdot)$ thus contains pure terms in $\left\{A_{i}\right\}$ and does not vanish for $A_{j}=0(j \notin \mathscr{M})$; hence $\mathscr{C}$ is not a class of motion.

Remark 1: Theorem 3 holds for generic systems. However, when some nonlinearities are lacking, some classes of motion can exist in addition to that admitted by Eq. (18) even if the condition is violated. This occurs when, in the absence of generator terms, the generated terms contain only products $A_{j} \overline{A_{j}}(j \notin \mathscr{M})$ and no products $A_{i} \overline{A_{i}}(i \in \mathscr{M})$. As an example, the resonance condition $\omega_{2}=2 \omega_{1}$ does not admit the 1-class $\left\{A_{1}\right\}$ according to Eq. (18) and to the fact that the $A_{2}$-equation reads $A_{2}^{\prime}=\mathscr{L}_{2}\left(A_{1}^{2}\right)$. However, if the generator term $A_{1}^{2}$ is absent from this equation, it generates the terms $A_{1}^{2} A_{1} \overline{A_{1}}, A_{1}^{2} A_{2} \overline{A_{2}}$ (remember Eq. (12)). If the first of these terms is also absent, then $A_{2}^{\prime}=\mathscr{L}_{2}\left(A_{1}^{2} A_{2} \overline{A_{2}}\right)$ and $\mathscr{L}_{2}=0$ if $A_{2}=0$; therefore $\left\{A_{1}\right\}$ is a 1-class for the non-generic system as an exception to Theorem 3 . In contrast, the 1-class $\left\{A_{2}\right\}$ is admitted by Eq. (18), since $A_{1}^{\prime}=\mathscr{L}_{1}\left(A_{2} \overline{A_{1}}\right)$. Even if the generator terms were absent, any generated terms $A_{1} A_{2} \bar{A}_{1}^{2}, A_{2}^{2} \overline{A_{1}} \overline{A_{2}}$ would vanish when $A_{1}=0$.

Remark 2: From Theorem 3, it follows that the resonance conditions (1) in which all the coeffcients $\left|k_{s n}\right| \neq 1$ are unessential to classes in evaluation. Thus, for example, resonance conditions proportional to a given condition (e.g., $2 \omega_{1}=2 \omega_{2}$ ) can be ignored in the analysis.

Theorem 3 suggests the following algorithm to evaluate all the classes of motion of a (generic) multiresonant system:

Algorithm 1: RCM (Resonant Coefficient Method): (1) The table $\left[k_{s n}\right](n=0,1, \ldots, N)$ of the resonance coefficients is built up by ignoring proportional conditions; (2) a set of $M$ columns (always including column $k_{s 0}$ ) is cancelled: if the remaining part of the table does not contain the string $(0, \ldots, 0, \pm 1,0, \ldots, 0)$ on any rows, then the $M$ amplitudes associated with the $M$ columns are an $M$-class of motion for the system; (3) all the combinations of $M$ columns are tried and step (2) is repeated.

As an example of the RCM, let us again consider Eq. (14), with $\omega_{0}=0$ for the sake of simplicity. It is $N=5, R=3$ and $Q=1$ up to the third order: by ignoring the proportional condition $2 \omega_{3}=2 \omega_{4}$, the table of the resonance coefficients reads: 


\begin{tabular}{ccccc}
$\omega_{1}$ & $\omega_{2}$ & $\omega_{3}$ & $\omega_{4}$ & $\omega_{5}$ \\
\hline 1 & -2 & 0 & 0 & 0 \\
0 & 1 & -1 & 0 & -2 \\
0 & 0 & 1 & -1 & 0 \\
0 & 1 & 0 & -1 & -2
\end{tabular}

By canceling the first column no strings are found containing a unique digit \pm 1 and all zeros, and therefore $\left\{A_{1}\right\}$ is a 1-class. In contrast, if the second column is canceled, such a string is found on the first row, and $\left\{A_{2}\right\}$ is therefore not a 1-class. Similarly, $\left\{A_{1}, A_{2}\right\}$ is a 2-class, but $\left\{A_{1}, A_{3}\right\}$ is not since the third resonance condition inhibits it. By repeatedly applying the test, the following classes of motion are found: $\left\{A_{1}\right\},\left\{A_{5}\right\} ;\left\{A_{1}, A_{2}\right\},\left\{A_{1}, A_{5}\right\},\left\{A_{3}, A_{4}\right\}$; $\left\{A_{1}, A_{3}, A_{4}\right\} ;\left\{A_{1}, A_{2}, A_{3}, A_{4}\right\}$, in addition, of course, to the complete class.

\section{Discussion}

\subsection{Degree of constraint of resonance conditions}

It was noted in Sect. 3.2 that if no resonance conditions are present, all the combinations among the amplitudes are classes of motion. Moreover, the RCM has shown how any resonance condition works as a constraint on such classes by inhibiting some of them and allowing others. Only classes that are not constrained by any resonance condition are admitted by the multiresonant system. Therefore, the higher the number of resonance conditions, the lower the number of classes of motion admitted.

Besides giving an interesting meaning to the resonance conditions, the above considerations open a new question: given that the constraints associated with each resonance condition are generally not distinct, is it possible to find a reduced set of 'principal' resonance conditions still able to furnish all the classes of motion of the complete system? In other words, is it possible to ignore some of the resonance conditions, in order to simplify the search for the classes? The question is still unresolved, since the authors were unable to provide an exhaustive answer to it; however, some indication of the efforts made to address the problem is given below.

Firstly, it was conjectured that, since only $R$ of $S$ conditions are independent, there should exist just $R$ principal conditions. Example (19) would corroborate this hypothesis: if the fourth (dependent) condition is ignored, the first three rows still furnish the correct information. However, not all the sets of $R=3$ independent conditions have this property. Indeed, if the third row is ignored instead of the fourth, some information is lost, e.g., $\left\{A_{1}, A_{3}\right\}$ would erroneously be admitted by the reduced set. However, the previous conjecture revealed itself to be false, as the following example clarifies. Let us consider $N=5$ frequencies involved in the following $R=2$ resonances: $\omega_{1}=\omega_{4}+2 \omega_{5}, \omega_{3}+3 \omega_{5}=\omega_{2}$. Up to order four, only one dependent condition $\omega_{1}+\omega_{3}+\omega_{5}=\omega_{2}+\omega_{4}$ (sum of the previous two) is found, so that the table $\left[k_{s n}\right]$ reads:

\begin{tabular}{ccccc}
$\omega_{1}$ & $\omega_{2}$ & $\omega_{3}$ & $\omega_{4}$ & $\omega_{5}$ \\
\hline 1 & 0 & 0 & -1 & -2 \\
0 & -1 & 1 & 0 & 3 \\
1 & -1 & 1 & -1 & 1
\end{tabular}


It is easy to see that each row entails a constraint which is not included in the other two; in particular: the first condition inhibits the 2-class $\left\{A_{4}, A_{5}\right\}$, the second $\left\{A_{3}, A_{5}\right\}$, and the third the 4 -class $\left\{A_{1}, A_{2}, A_{3}, A_{4}\right\}$, that would instead be admitted by the other two resonances. Hence, all three conditions are necessary to evaluate the class, notwithstanding $R=2$.

Although general rules cannot be given here, a useful strategy to choose possible 'principal' resonances is the following. A constraint degree $c_{s}$ is defined as the number of classes inhibited by the $s$-th condition and a hierarchy among the resonances is established: the higher $c_{s}$, the higher the level in the hierarchy of the $s$-th resonance. Although there is no guarantee that the conditions of the lowest level are inessential, there is a strong probability that the resonances of highest level contain all the independent constraints.

To evaluate $c_{s}$, use is made of Theorem 3. Accordingly, $c_{s}$ is equal to the number of strings that can be drawn among the $k_{s n}$ coefficients (by excluding $k_{s 0}$ ), containing a unique \pm 1 digit and $0,1,2, \ldots$ zero digits. By using $n_{1}$ and $n_{0}$ to denote the number of coefficients $k_{s n}(n \neq 0)$ equal to \pm 1 and 0 , respectively, and remembering that the number of combinations among $n_{0}$ objects is equal to $2^{n_{0}}-1$, it follows that:

$c_{s}=n_{1} 2^{n_{0}}$.

For example, by applying Eq. (21) to the resonance conditions (19), the following constraint degrees are found, ordinately: $c_{s}=8,8,16,8$. Thus, the higher level resonance is $\omega_{3}=\omega_{4}$, since it inhibits sixteen classes of motion, while the remaining are of the same level, with eight classes inhibited. It is possible to check that the highest-level condition contains constraints that are different from the lowest (e.g., it destroys the 1-class $\left\{A_{3}\right\}$ ), so that it cannot be ignored; moreover, the second and fourth conditions bring identical new constraints in relation to the others, so that one of them can be ignored. As a second example, the constraint degrees of the resonances (20) are $c_{s}=8,8,5$, respectively. In this case, not withstanding the third condition is of the lowest level, it cannot be ignored, as previously discussed. However, it has been checked in this example that conditions of higher-order $K_{s}$ have lower $c_{s}$ and do not introduce new constraints. Therefore, it is believed that the constraint degree criterion is helpful in selecting the most significant resonance conditions.

\subsection{Numerical investigation of the robustness of the class of motion}

To evaluate the resonances appearing in higher-order approximations of the AME, a system is considered in which $\omega_{1}=1, \omega_{2}=5$ and $\omega_{3}=8$. The following order-4 primary resonances are encountered:

$\omega_{3}=3 \omega_{1}+\omega_{2}, \quad \omega_{3}=2 \omega_{2}-2 \omega_{1}$,

while the following higher-order resonances exist at order five:

$\omega_{2}=5 \omega_{1}, \quad \omega_{1}=2 \omega_{3}-3 \omega_{2}$.

By using the RCM it is possible to predict that the 1-class $\left\{A_{1}\right\}$ and the 2-class $\left\{A_{1}, A_{3}\right\}$ admitted by the primary resonances (22) disappear if the higher-order resonances (23) are considered. A numerical investigation was performed to analyze how higher-order resonant terms destroy the lower-order class. Let us consider the following equations of motion:

$\ddot{q}_{i}+\omega_{i}^{2} q_{i}=\alpha \sum_{l_{1}, m_{1}, n_{1}} q_{1}^{l_{1}} q_{2}^{m_{1}} q_{3}^{n_{1}}+\beta \sum_{l_{2}, m_{2}, n_{2}} q_{1}^{l_{2}} q_{2}^{m_{2}} q_{3}^{n_{2}}$, 

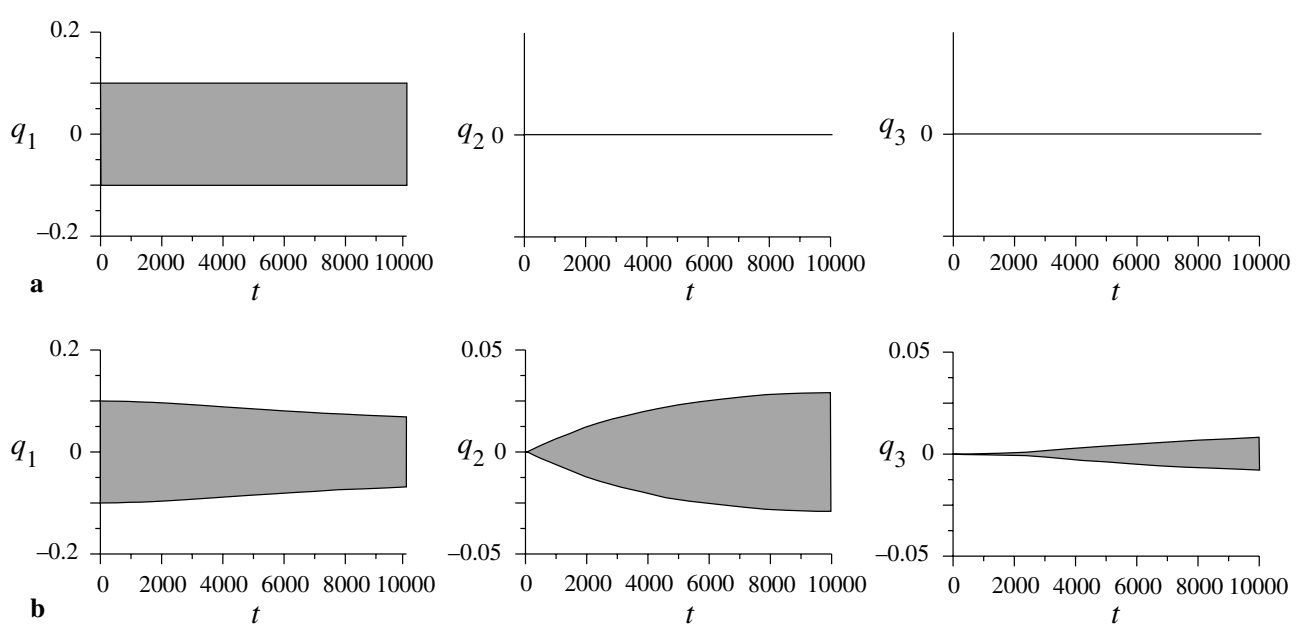

Fig. 1. 1 Class motion $\left\{A_{1}\right\}$ : a order 5 terms in Eq. (24) neglected; b order 5 terms in Eq. (24) retained
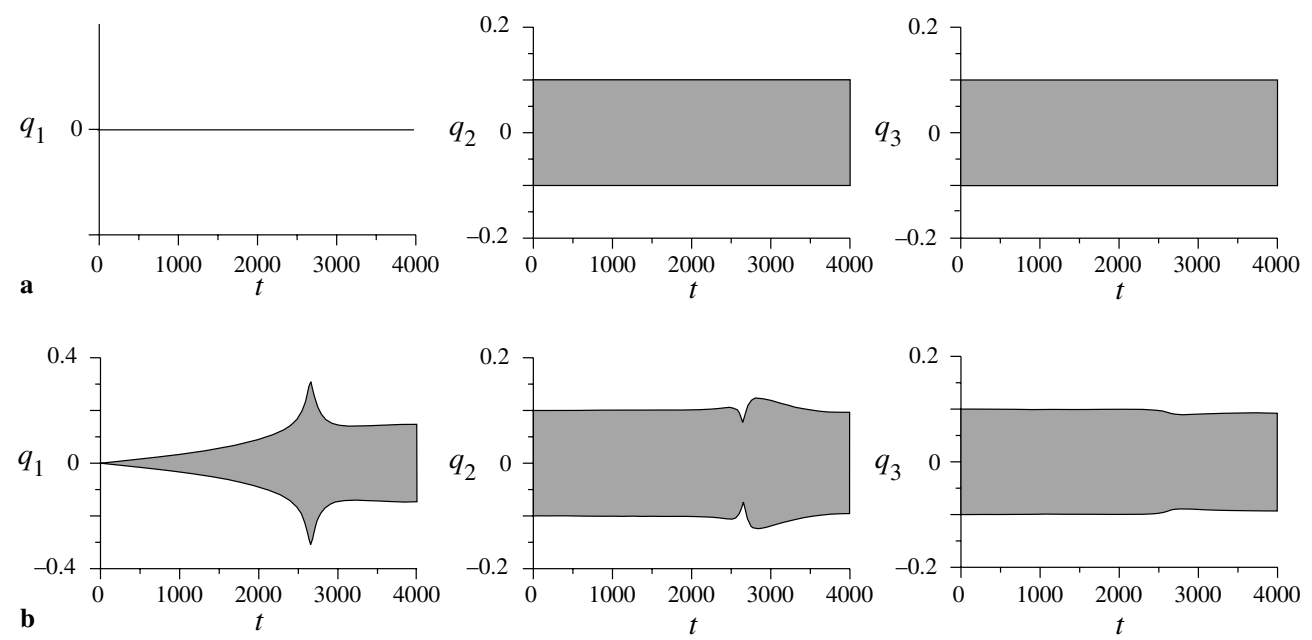

Fig. 2. 2 Class motion $\left\{A_{1}, A_{2}\right\}$ : a order 5 terms in Eq. (24) neglected; b order 5 terms in Eq. (24) retained

with $(i=1,2,3),\left(l_{1}+m_{1}+n_{1}=4\right),\left(l_{2}+m_{2}+n_{2}=5\right)$ and in which only the nonlinear resonant terms are retained; moreover, $\alpha=10$ and $\beta=100$ are assumed. A 1-Class $\left\{A_{1}\right\}$ motion is shown in Fig. 1a when order-5 terms are omitted or considered (Fig. 1b). A 2-Class $\left\{A_{2}, A_{3}\right\}$ motion is also shown in Fig. 2a and $2 \mathrm{~b}$ when terms of order 5 are neglected or taken into account, respectively. In both cases, when higher-order resonance terms are considered, the classes of motion remain almost unchanged for a long time until the out-of-class components become meaningful. In summary, it is confirmed that higher-order resonances could destroy some classes of motion, though such a process is very slow. 


\section{Conclusions}

Classes of motion of general multiresonant systems, free or harmonically forced, have been qualitatively analyzed and an algorithm to find all of them presented. To apply the method, only knowledge of the resonance conditions is required, and no preliminary construction of the Amplitude Modulation Equations (AMEs) is necessary. The following conclusions are drawn:

(1) The resonance conditions existing in a dynamical system among free and forcing frequencies can be classified as independent and dependent conditions. The former have maximum rank, while the latter are linearly dependent on them. However, dependent conditions are also important in the equations of motion, since they essentially give rise to new terms in the AMEs. Both the analysis and the design problem of a system have been addressed and a numerical algorithm has been illustrated: in the former, given the frequencies, all the independent and dependent resonance conditions are sought up to a given order; in the latter, a set of frequencies is found satisfying the desired resonance conditions and no more. A numerical example of the design problem has also been given.

(2) General rules for calculating up to any order the type of the terms entering the AMEs have been determined. These make possible to write (to within the coefficients) the AMEs of a general system without performing any perturbation analysis. Different classes of terms have been classified: (a) improper resonant terms and (b) primary and (c) secondary proper resonant terms. Improper terms do not depend on the resonance conditions and are present in the AMEs even if the system is nonresonant. Proper terms, in contrast, depend directly on resonance and appear in the perturbation scheme at the same order as the resonance (primary terms) or at higher orders (secondary terms).

(3) Classes of motion have been defined as invariant subspaces of the amplitude space. It has been proved that this existence depends only on the primary resonant terms, the remaining terms having only a quantitative effect on the motion. Reference can therefore be made to reduced equations if only the qualitative aspects are of interest.

(4) It is found that higher-order asymptotic solutions do not modify the classes of motion of a system once the primary terms have appeared in the perturbation procedure, unless other resonances are encountered. A numerical analysis has been performed on an example to check this result.

(5) A simple and efficient algorithm (the Resonance Coefficient Method, RCM) has been implemented, that is able to find all the existing classes of motion simply by analyzing the resonance conditions. The method works well for general systems, for which all the nonlinear terms are assumed to be different from zero. Particular systems, for which some terms may be absent, usually possess more classes of motion than general systems.

(6) The resonance conditions have been interpreted as constraint relations for the classes of motion, since they prevent the existence of some of them. If a system is nonresonant, all the classes are admitted; the larger the number of resonances, the smaller the number of classes. A constraint degree has been attributed to each resonance, equal to the number of classes it inhibits. The index allows the resonances to be ordered hierarchically: resonances with a lower degree of constraint are usually unessential to the evaluation of the classes, but general rules are not yet available.

(7) Two aspects of the work are worthy of further analysis: (a) to investigate if combinations of classes of motion give an approximate description of more complex motions e.g., along the lines of the studies on nonlinear normal modes [7]; (b) to implement an identification procedure for the coefficients of the AMEs, in order to build up an analytical model for a real system, based on experimental observations. 


\section{Appendix A}

Algorithms for detecting resonance conditions

Two algorithms are described to solve the following problems, discussed in Sect. 2.1.

Problem 1 (Analysis problem): Given a set of frequencies $\omega:=\left\{\omega_{0}, \omega_{1} \ldots, \omega_{N}\right\}$, let us determine the resonance conditions (1) of orders $K_{s} \leq K_{\max }$.

Problem 2 (Design problem): Given a set of $R \leq N_{0}-1$ homogeneous independent conditions of orders $K_{s} \leq K_{\max }$ involving the frequencies $\omega:=\left\{\omega_{0}, \omega_{1}, \ldots, \omega_{N}\right\}$, let us determine: (a) the possible values of $\omega$ for which no other independent conditions exist up to order $K_{\max }$ (in addition to the given ones); (b) all the dependent conditions existing up to order $K_{\max }$.

In the former, $N_{0}:=N$ if $\omega_{0}=0$ (i.e., if all the resonances are of internal type) or $N_{0}:=N+1$ if $\omega_{0} \neq 0$ (i.e., if one or more resonances are external).

In Problem 1, a prefixed minimum detuning must be chosen, beyond which a combination of frequencies should be considered as non-resonant. However, care must be taken to avoid losing any dependent conditions, when linear combinations of the independent conditions cause an increase in the detuning. To solve Problem 1, the following algorithm can be applied.

Algorithm 2 (Analysis problem): (1) All the sets $\left\{k_{s n}\left|\sum_{n}^{N}{ }_{0}\right| k_{s n} \mid-1=K_{s}\right\}$ are built up (by discarding the set with an opposite sign) with $k_{s n}=0, \pm 1, \pm 2, \ldots, \pm K_{s n}$ and $K_{s}=1,2, \ldots, K_{\max }$; (2) the combinations $\sigma_{s}:=\sum_{n}^{N}{ }_{0} k_{s n} \omega_{n}$ are evaluated and retained as resonant only if $\sigma_{s}<<1$; (3) the $S \times N_{0}$ matrix $\left[k_{s n}\right]$ of the resonance coefficient is built up and the number $R:=\operatorname{rank}\left[k_{s n}\right]$ is evaluated; (4) Eqs. (1) are rearranged as:

$\sum_{n 0}^{N} k_{s n}^{i} \omega_{n}=\sigma_{s}^{i} \quad s=1,2, \ldots, R$,
$\sum_{n 0}^{N} k_{s n}^{d} \omega_{n}=\sigma_{s}^{d} \quad s=R+1, \ldots, S$,

where the first $R$ equations are the independent conditions, and the remaining $Q:=S-R$ equations the dependent conditions.

Since Eqs. (A.1.2) are linearly dependent on Eqs. (A.1.1.), it follows that

$k_{s n}^{d}=\sum_{r}^{R} \alpha_{s r} k_{r n}^{i}, \quad \sigma_{s}^{d}=\sum_{r 1}^{R} \alpha_{s r} \sigma_{r}^{i} \quad s=R+1, \ldots, S, \quad \alpha_{s r} \in \mathbb{Q}$.

It is also easy to prove that, since by hypothesis the given frequencies $\omega$ are different from zero, the number of independent conditions is

$R \leq N_{0}-1$,

i.e., the case $R=N_{0}$ must be excluded. To prove this, let us first assume that the detunings $\sigma_{s}^{i}$ are all zero (perfect resonances). Then, if $R=N_{0}$, Eqs. (A.1.1) would be verified only by $\boldsymbol{\omega}=\mathbf{0}$, contrary to the hypothesis. These results also hold when some or all the $\sigma_{s}^{i}$ are different from zero, since, as they are small, the solutions of Eqs. (A.1.1) are perturbations of the perfect resonance equations. 
To solve Problem 2, the following strategy is adopted. If $R=N_{0}-1$, the given $R$ equations $\sum_{n}^{N} k_{m} \omega_{n}=0 \quad r=1,2, \ldots, R$

are sufficient to determine the vector $\omega$ to within one component, to be fixed arbitrarily (normalization condition). Since the maximum rank of the resonance coefficients cannot exceed $N_{0}-1$, no other independent conditions exist in this case. If $R<N_{0}-1$, then Eqs. (A.4) plus the normalization condition determine only $R+1$ "constrained" frequencies $\omega_{c}$ as linear functions of $N_{0}-R-1$ "free" frequencies $\omega_{f}$, so that $\omega=\left\{\omega_{c}\left(\omega_{f}\right), \omega_{f}\right\}$. However, the free frequencies cannot be chosen arbitrarily, since they could satisfy additional undesired resonance conditions. Their resonant values (i.e., the values they should not assume) are determined when $\omega$ satisfies all the independent sets of $R+1$ equations obtained by appending to Eqs. (A.4) any of the resonance conditions which increase the rank, i.e.,

$\sum_{n}^{N} k_{p n} \omega_{n}=0 p=R+1, R+2, \ldots ; \forall k_{p n} \mid\left(\sum_{n}^{N}\left|k_{p n}\right|-1 \leq K_{\max }, \operatorname{rank}\left[\begin{array}{c}{\left[k_{r n}\right]} \\ k_{p n}\end{array}\right]=R+1\right)$.

When these equations are solved, resonant subspaces of codimension 1 in the $\omega_{f}$-space are determined. Finally, the required set $\omega$ must be selected in such a way that its projection $\omega_{f}$ is sufficiently far from all the resonant subspaces determined. It is worth noticing that if more than one independent equation is appended to Eqs. (A.4), other resonant subspaces of higher codimension are found. However, they are enclosed in the larger subspaces, since they are evaluated by adding more conditions to the previous ones. They thus make no contribution to evaluating the resonant subspaces.

The procedure is summarized by the following

Algorithm 3 (Design problem): (1) all the sets $\left\{k_{s n}\right\}(s=1,2, \ldots)$ described in Algorithm 2 are built up, and from them, the $R$ given sets $\left\{k_{m}\right\}$ are extracted; (2) a normalization condition, e.g. $\omega_{1}=\omega_{1}^{*}$, is appended to Eqs. (A.4) and the system solved in terms of $N_{0}-R-1$ free frequencies $\omega_{f}$; (3) the solution is substituted into the linear equation $\sum_{n}^{N}{ }_{0} k_{p n} \omega_{n}=0$, with $\left\{k_{p n}\right\}$ in turn taken from $\left\{k_{s 1}\right\}$; (4) if Eq. (A.5) is identically satisfied, then $\left\{k_{p n}\right\}$ is a set of coefficients of a dependent condition; (5) if it is not identically satisfied, then it is solved for the
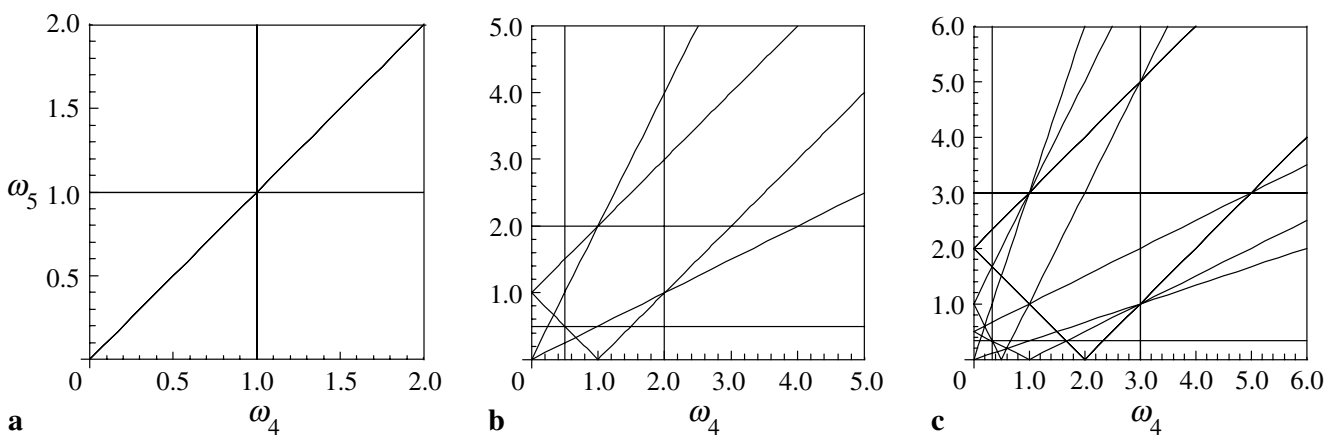

Fig. 3. Resonant subspace in the $\omega_{f}$ plane for the example of Eq. (A.6): a $K=1 ; \mathbf{b} K=2$; $⿱$ c $K=3$ 
free frequencies, and a subspace is found in the $\omega_{f}$ space; (6) at the end of the procedure, values for $\omega_{f}$ must be chosen far from the resonant ones, from which the remaining $\omega_{c}$ frequencies are determined.

As an illustrative example, let us design a system having $N=N_{0}=5$ frequencies $\omega=\left\{\omega_{1}, \ldots, \omega_{5}\right\}^{T}$ among which only $R=2$ independent resonance conditions must hold, namely:

$\omega_{1}=\omega_{3}, \quad \omega_{2}=\omega_{3}$.

By choosing for example $\omega_{1}=1$ (normalization condition), Eqs. (A.6) furnish the constrained frequencies $\omega_{c}=\left\{\omega_{1}, \omega_{2}, \omega_{3}\right\}^{T}=\{1,1,1\}^{T}$; therefore the free frequencies are $\omega_{f}=\left\{\omega_{4}, \omega_{5}\right\}^{T}$. By taking $K=1$, the independent resonance conditions $\omega_{i}=\omega_{j}(i \neq j)$ are found, including Eqs. (A.6). However, by accounting for the known values of $\omega_{c}$ and discarding the identities, they reduce to three conditions involving the frequencies $\omega_{f}$, namely: $\omega_{4}=1, \omega_{5}=1, \omega_{4}=\omega_{5}$. These latter describe on the $\left(\omega_{4}, \omega_{5}\right)$-plane the straight lines of Fig. 3a, which are locations of resonant frequencies. A pair $\left(\omega_{4}, \omega_{5}\right)$ far from these locations must be taken if Eqs. (A.6) are to be the unique independent resonance conditions of order 1 . However, if $K>1$ is taken, more resonance conditions are encountered and more resonant locations are found on the $\left(\omega_{4}, \omega_{5}\right)$ plane. Figure $3 \mathrm{~b}$ and $\mathrm{c}$ shows these for $K=2$ and $K=3$, for which nine and fifteen new resonant locations are found, respectively, in addition to the three first-order ones.

\section{Acknowledgement}

This work was supported by MURST (COFIN 01-03).

\section{References}

[1] Nayfeh, A. H., Balachandran, B.: Applied nonlinear dynamics. New York: Wiley 1995.

[2] Troger, H., Steindl, A.: Nonlinear stability and bifurcation theory. Wien New York: Springer 1991.

[3] Luongo, A., Di Egidio, A., Paolone, A.: On the proper form of the amplitude modulation equations for resonant system. Nonlinear Dynamics 27, 237-254 (2002).

[4] Nayfeh, A. H., Mook, D. T.: Nonlinear oscillations. New York: Wiley 1979.

[5] Nayfeh, A. H.: Topical course on nonlinear dynamics. Società Italiana di Fisica, Santa Margherita di Pula. Sardinia, Perturbation Methods in Nonlinear Dynamics, 1985.

[6] Vakakis, A. F., Mikhlin, L. I., Yu, V., Pilipchuck, M., Zevin, A. A.: Normal modes and localization in nonlinear systems. New York: Wiley 1996.

[7] Shaw, S. W., Pierre, C.: Normal modes for nonlinear vibratory systems. J. Sound Vibr. 164, 85124 (1993).

Authors' addresses: A. Luongo and A. Di Egidio, Dipartimento di Ingegneria delle Strutture, delle Acque e del Terreno, Università di L'Aquila, Monteluco di Roio (L'Aquila) 67040, Italy (E-mail: luongo@ing.univaq.it); A. Paolone, Dipartimento di Ingegneria Strutturale e Geotecnica, Università degli Studi di Roma La Sapienza, Via Eudossiana, 18, Roma 00184, Italy (E-mail: achille.paolone@uniroma1.it) 